\title{
Quantum transport through one-dimensional aluminum wires
}

\author{
Inder P. Batra ${ }^{\text {a) }}$ and Prasenjit Sen \\ Department of Physics, University of Illinois at Chicago, Chicago, Illinois 60607-7059 \\ S. Ciraci \\ Department of Physics, Bilkent University, Bilkent, Ankara 06533, Turkey
}

(Received 29 October 2001; accepted 18 February 2002)

\begin{abstract}
Quantum conductance in narrow channels has been well understood by using the two-dimensional electron gas, a model system which has been realized in semiconductor heterojunctions. An essential property of this electron gas is its ability to support a constriction of width comparable to the Fermi wavelength, a property not shared by even thin metal films. The advent of scanning tunneling microscope has made possible the fabrication of metallic wires of atomic widths. We investigate one-dimensional wires consisting of aluminum atoms, to be specific. Using the first-principles density functional calculations, we obtain the optimal structures and report the bonding as deduced from the charge density analysis. With the calculated electronic structure in hand, we discussed the quantum ballistic transport using channel capacity arguments motivated by the Heisenberg's uncertainty principle. By comparing our results with the detailed pioneering calculations by Lang, we inferred an average value for channel transmitivity and touched upon material specific contact resistance. Finally, the validity of the Wiedemann-Franz law in the quantum domain is established by studying thermal conductance in nanowires. (C) 2002 American Vacuum Society.
\end{abstract}

[DOI: $10.1116 / 1.1468659]$

\section{INTRODUCTION}

Advances in nanofabrication and the emerging novel results are in the domain where concepts in quantum physics are required for their explanation. Earlier, much of the work on conductance quantization was carried out on GaAsAlGaAs heterostructures supporting a thin conducting layer at the interface. ${ }^{1,2}$ The reason is that for the quantization effects to be observable, the constriction width must be in the range of the Fermi wave length $\lambda_{F}$. The Fermi wavelength of the electrons, in the semiconductor heterostructures can be estimated from the two-dimensional charge density $\left(\sim 10^{15} / \mathrm{m}^{2}\right)$, and it turns out to be several hundred $\AA$. $\mathrm{A}$ constriction of this width can be easily created by applying a negative confining gate voltage in one direction. The carriers are then free to move only in the orthogonal direction, making the system quasi-one dimensional (1D). By changing the value of the gate voltage, the constriction width can be varied and hence the conductance quantization can be studied as a function of the constriction width. Also, for ballistic transport, the elastic mean free path should be longer than the constriction length, a condition easily met in semiconductor heterostructures. That is the reason for the first confirmation of conductance quantization coming from semiconductor heterostructures.

For thin metal films, the Fermi wavelength is about $1 \AA$. If any quantization effects are to be studied in the metallic systems, one must first create contacts of atomic dimensions. Ordinary metal films even of submicron widths $(w)$ fail to show any quantization conductance jumps ${ }^{3-5}$ because $\lambda_{F}$ $\ll w$. The discovery of scanning tunneling microscope by Binning et $a l .{ }^{6}$ changed all this. One can now routinely pro-

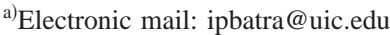

duce atomic size wires. ${ }^{7,8}$ Stable gold monoatomic chains suspended between two gold electrodes have also been obtained by stretching gold nanowires. ${ }^{9,10}$

The stable structural arrangement of atoms in 1D nanowires have been recently published for many different types of atomic chains. The first-principles calculations for Au by Portal et al. ${ }^{11}$ showed that infinite, as well as finite, gold atomic wires between two gold electrodes favor the planar zigzag geometry. In a more recent comparative study, ${ }^{12} \mathrm{Au}$, $\mathrm{Cu}, \mathrm{Ca}$, and $\mathrm{K}$ infinite chains were found to form planar zigzag structures with equilateral triangular geometry; only the Au chain has a second zigzag structure with a wide bond angle $\alpha=131^{\circ}$. All these atoms are similar because of their $s$-type outermost valence orbitals.

We expect a chain of aluminum atoms to display a different behavior since an $\mathrm{Al}$ atom has $3 s^{2} 3 p^{1}$ valency. Our focus here primarily is on the electronic conductance through $1 \mathrm{D}$ aluminum wires. Structural arrangements of atoms is a prerequisite for such an analysis. In this connection, we will briefly review our recent findings ${ }^{13}$ about geometrical arrangements and provide additional results. The expected conductance values for $\mathrm{Al}$ nanowires will then be discussed.

We will remind the readers that the conductance quantization value ${ }^{14,15}$ or the maximum channel capacity can be derived directly from the Heisenberg's uncertainty principle. But more significantly, some justification will be provided for this derivation. We draw upon the pioneering results obtained by Lang ${ }^{16,17}$ for $\mathrm{Al}$ and $\mathrm{Na}$ atomic wires consisting of only a few atoms in length and point to material specific contact resistance effects embodied in his calculations. His results enable us to deduce an approximate value for an average channel transmitivity. In Sec. IV, the validity of the Wiedemann-Franz law in the quantum domain is established 


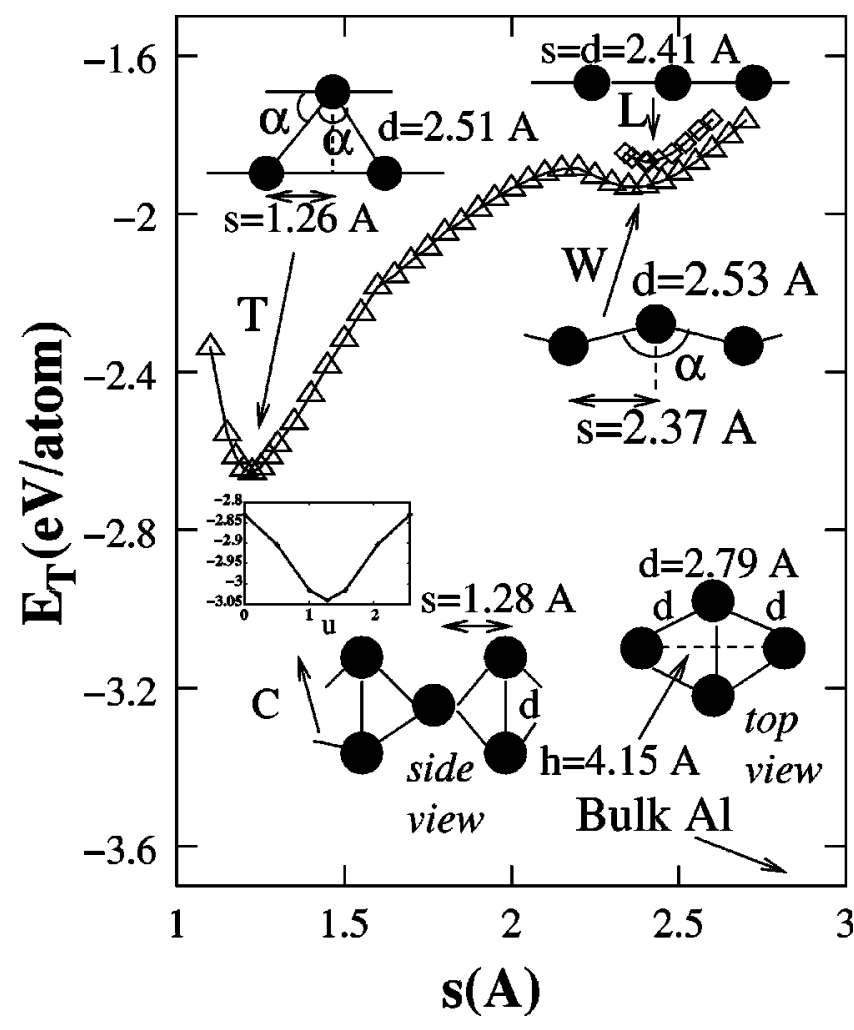

FIG. 1. Calculated total energy $E_{T}\left(=-E_{C}\right)$ of infinite $\mathrm{Al}$ chains with linear, planar (wide-angle and equilateral triangles) and nonplanar (cross) structures. Calculated cohesive energy of bulk Al is indicated by an arrow. Relevant structural parameters, bond length $d$, bond angle $\alpha$, etc., for the equilateral triangular $(T)$, wide-angle $(W)$, and nonplanar cross $(C)$ structures are given as insets. Another inset shows energy variation of the $C$ structure for moving one of the dumbbells along the length of the infinite chain while keeping the lattice constant fixed in that direction. The distance between the two dumbbells is $u$, the lattice constant was fixed at $2.56 \AA$.

by studying thermal conductance in nanowires. A short summary of our results is presented in the closing section.

\section{GEOMETRICAL AND ELECTRONIC STRUCTURE OF 1D ALUMINUM WIRES}

All our calculations were performed in the framework of the density functional theory in conjunction with ultrasoft Vanderbilt type pseudopotentials. ${ }^{18}$ The well known VASP code $^{19}$ was employed for the numerical calculations. The variation of the total energy $E_{T}$, of the atomic $\mathrm{Al}$ chain for the fully relaxed Al chains are shown in Fig. 1. The energy was obtained from the relation, $E_{T}=[E(\mathrm{Al}$-wire $)$ $\left.-n E_{\mathrm{Al}}\right] / n$, where the total energy of $E(\mathrm{Al}$-wire) was calculated using $n$ atoms in the unit cell, and $E_{\mathrm{Al}}$ is the $\mathrm{Al}$ atomic energy. The geometry of these structures is shown by insets. The $z$ axis was taken along the Al chain axis, and $y$ axis $(x$ axis) is perpendicular to (in) the plane of zigzag structure. The cohesive energy $E_{C}=-E_{T}$.

The zigzag geometry displays two minima; one occurs at $s=1.26 \AA$ and has cohesive energy $E_{C}=2.65 \mathrm{eV} /$ atom; the other has shallow minimum and occurs at $s=2.37 \AA$ with cohesive energy $E_{C}=1.92 \mathrm{eV} /$ atom. The high cohesive energy zigzag structure (specified as $T$ ) having the bond length $d=2.51 \AA$, and the bond angle $\alpha \sim 60^{\circ}$ forms equilateral triangles. This geometry allows for four nearest neighbors. The low cohesive energy zigzag structure (specified as $W$ ) has $d=2.53 \AA$ and wide bond angle $\alpha \sim 139^{\circ}$, and allows for only two nearest neighbors with bonds slightly larger than those of the $T$ structure. It is worth noting that cluster calculations for $\mathrm{Al}$ have also reported ${ }^{20}$ that the most stable isomers for $\mathrm{Al}_{3}$ form an equilateral triangle. The calculated bond lengths in the range of 2.3-2.6 $\AA$ are rather similar to our value for the $T$ structure. No stable cluster corresponding to the local arrangement in our $W$ structure has been reported. The reason for this is that in an infinite structure, each $\mathrm{Al}$ atom can form two $\sigma$ bonds and one (weak) $\pi$ bond. In a wide bond angle $\mathrm{Al}_{3}$ cluster, there is the cohesion energy loss corresponding to at least one $\sigma$ bond. The minimum energy of the linear infinite chain structure $\left(\alpha=180^{\circ}\right.$ and denoted as $L$ ) has relatively short bond length, $d=s=2.41 \AA$. It is $\sim 0.05 \mathrm{eV}$ above the minimum energy of the $W$ structure and has cohesive energy $E_{C}=1.85 \mathrm{eV} /$ atom. Our results for $\mathrm{Al}$ are similar to those reported for $\mathrm{Au}$ atomic chain. ${ }^{12}$

Among the planar 1D structures, the lowest energy is the equilateral $T$ structure. This structure can be viewed as if two parallel linear chains with an interchain distance $(s \sqrt{3})$ of $2.17 \AA$ are displaced by $s$ along the $z$ direction. This chain is two-atom wide along the $x$ direction (periodic along $z$ with a period of $2.52 \AA$ ) and is confined to the $z x$ plane. An important lower energy quasi-1D structure consists of two perpendicular dumbbells (A and B) for a total of four atoms per unit cell. This structure, labeled as $\mathrm{C}$ in the inset of Fig. 1, was first reported by Gülseren et al. ${ }^{21}$ The optimized length of dumbbell $\mathrm{B}(4.15 \AA)$ is calculated to be considerably longer than that of the dumbbell $\mathrm{A}(2.8 \AA)$ in the chain formed by the $\mathrm{ABABA} . .$. sequence of these dumbbells. An $\mathrm{Al}$ atom in dumbbell A forms a total of five bonds (one with the other atom in A, two with atoms in B above, and two with atoms in $\mathrm{B}$ below). Aluminum atom in dumbbell B forms only four nonplanar bonds since it has large intra-atomic distance.

The origin of the $C$ structure can be understood by looking at the local arrangement of the four atoms forming a parallelopiped in the $T$ structure. The atoms along the short diagonal can be viewed as forming a dumbbell of length 2.5 $\AA$, while those along the long diagonal separated by $4.34 \AA$ can be thought of as forming B. Since the triangles are equilateral in the $T$ structure, the "dumbbells" are mutually orthogonal to begin with. Upon energy optimization, the biggest effect is that these dumbbells become noncoplanar with an interplanar separation of $1.28 \AA$. The variation of the cohesive energy as a function of interplanar separation, $u$ is shown as an inset in Fig. 1. The maximum cohesive energy of the $C$ structure is $3.04 \mathrm{eV} /$ atom, is higher than the $T$ structure. It occurs when $u=s=1.28 \AA$ and the structure is periodic with a period equal to twice the interplanar separation. A qualitative explanation is due to the increased coordination.

One key question that must be addressed regarding the geometrical arrangement for 1D chains has to do with the Peierls distortion. Since all 1D metallic structures are ex- 
pected to distort to give a Peierls gap at the zone edge, the conductance would be simply zero. Yet, we see a finite conductance. The answer obviously lies in the magnitude of the gap. To test this, we investigated the linear geometry using four $\mathrm{Al}$ atoms per unit cell to allow for Peierls distortion. A linear chain of uniformly spaced $\mathrm{Al}$ atoms (interatomic distance $d$ ) has a doubly degenerate band which crosses $E_{F}$ at $\pm \pi / 4 d$ and is one quarter filled. Simply making the unit cell four times longer causes the doubly degenerate band to meet $E_{F}$ at the reduced zone edge. Due to folding, it is four-fold degenerate at this point and is half filled. Peierls condition is now precisely met and an internal rearrangement of the atoms should take place to change the crystal potential, which will lead to band splitting and a lowering of the total energy.

In fact, this is precisely what happens when an energy optimization calculation is carried out, allowing the atoms in the unit cell to move. A $4 d$ distortion where atoms located at $d$ and $3 d$ move away from the central $\mathrm{Al}$ atom (located at $2 d$ ) by small amounts is found to be the lowest energy arrangement. The energy of the distorted chain was $-7.4748 \mathrm{eV}$, only $10^{-4} \mathrm{eV}$ lower than the energy of the uniform chain. The band splitting at the zone edge due to this $4 d$ distortion was found to be $2 \times 10^{-4} \mathrm{eV}$ and is at the limit of our calculational accuracy. The conclusion is that the band splitting due to Peierls distortion is negligible, and under finite bias conditions, can be safely ignored.

The nature of the bonding for 1D structures was discussed earlier. ${ }^{13}$ It was found that in going from three-dimensional (3D) to 1D the bonds become directional in the 1D structures. The charge accumulation between atoms arises primarily from the $\sigma$ states (formed by $3 s+3 p_{z}$ orbitals). It was noted that the charge distributions of the $\mathrm{Al}-\mathrm{Al}$ bond differed from the corresponding charge distribution of Au zigzag structures as the latter showed no directional bonds. The $C$ structure, which is quasi-1D, starts to show some signs of delocalized charge distribution. Figure 2 presents the charge density contour plots along with a perspective of the $C$ structure. Charge distribution is shown in planes containing dumbbells A, B, and a plane half way in between. In the plane containing dumbbell $\mathrm{A}$, there is high charge density throughout, while in the plane of dumbbell $\mathrm{B}$, there is reduced charge density in the middle consistent with the absence of a bond between atoms forming B. All the bonds emerging from each atom are still clearly visible but we infer slight delocalization from charge contours in the middle of Fig. 2(c). This is reasonable, since with increased dimensionality, one should expect the emergence of metallic bonds.

The electronic band structure of Al zigzag chains can be understood by examining Fig. 3. Since the zigzag chains have two atoms per unit cell, we first show the folded bands for the uniform linear chain containing two atoms per unit cell, in Fig. 3(a). Recall that a linear chain of uniformly spaced $\mathrm{Al}$ atoms has a doubly degenerate band which crosses $E_{F}$ at $\pm \pi / 4 d$ and is one quarter filled. Doubling the size of the unit cell causes bands folding and the doubly degenerate band appears at the middle of reduced zone and is one half filled. The zone edge in this case lies at $Z=\pi / 2 d$. One no-
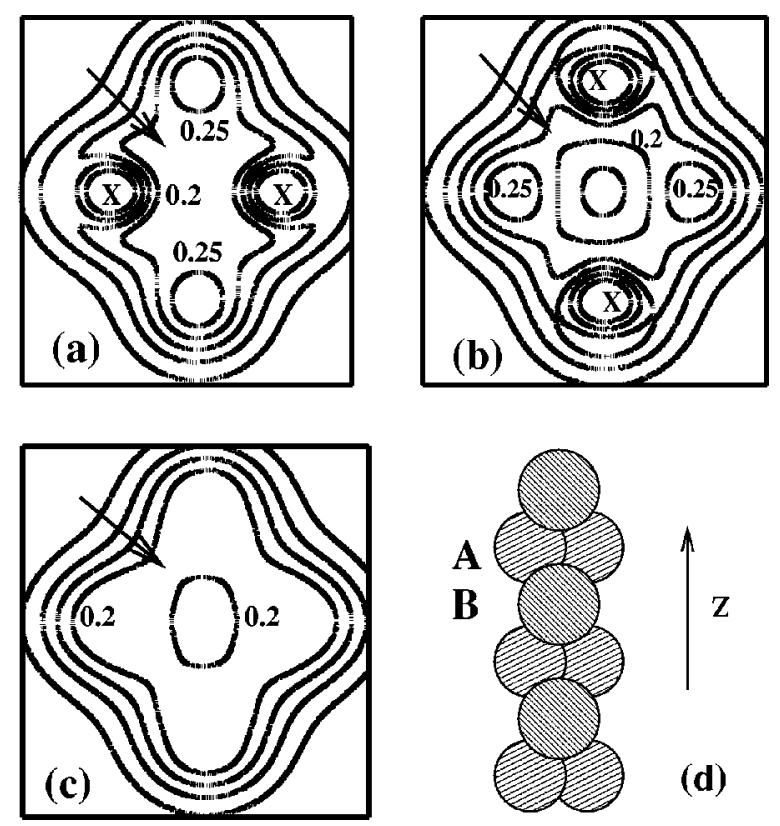

FIG. 2. Charge density contours for the $C$ structure in three different planes: (a) in the plane of the short dumbbell A, (b) in the plane of the long dumbbell B, and (c) halfway between the planes of the two dumbbells. Depletion of charge density in between the two atoms of dumbbell B in panel (b) is consistent with absence of any bond between them. Contours in panel (c) indicate more delocalization of charge as compared to the linear chain (d) perspective of the $\mathrm{C}$ chain labeling dumbbells $\mathrm{A}$ and $\mathrm{B}$.

tices from Fig. 3(a) that there are two filled $\sigma$-bands which arise from the $3 s+3 p_{z}$ valence orbitals. The $\pi$ band crossing the Fermi level is doubly degenerate since $3 p_{x}$ and $3 p_{y}$ are equivalent. This facilitates comparison with the bands of the zigzag structures, which actually contain two atoms per unit cell. The symmetry between $3 p_{x}$ and $3 p_{y}$ orbitals is broken in the zigzag structure, and hence the $\pi$ band is split. The $\pi$ bands for the $T$ structure [shown in Fig. 3(b)] are lowered, and the shape of the $\sigma$ bands changes significantly due to the equilateral triangular geometry. Two bands still

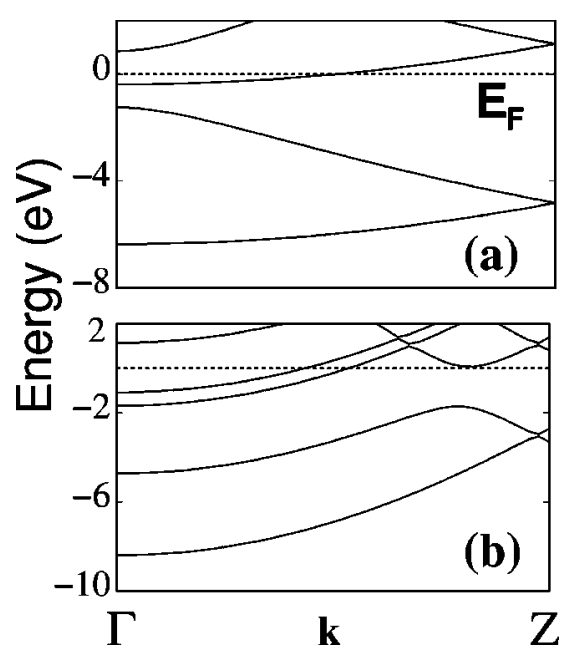

FIG. 3. Electronic band structures of (a) infinite linear chain with two atom unit cell and (b) $T$ structure. In both cases, two bands cross the Fermi level. 


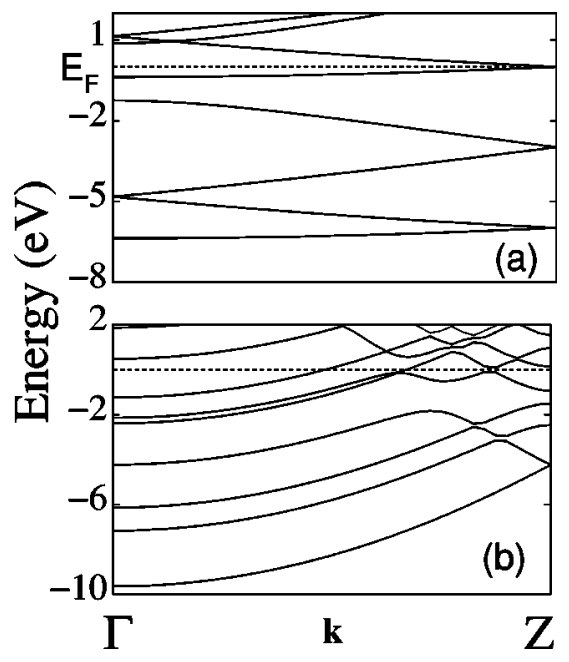

FIG. 4. Electronic band structures of (a) infinite linear chain with four atoms unit cell (b) $C$ structure with two dumbbells per unit cell.

cross the Fermi level. Brandbyge et al. ${ }^{22}$ report that three bands (one is doubly degenerate $p$ band) cross the Fermi level for an $\mathrm{Al}$ chain of uniform interatomic distance $2.86 \AA$. This has a profound effect on the quantum conductance value to be discussed later. Thus, it is important to obtain the proper optimized structures if one wants to compare to likely observed conductance values.

The bands for the $C$ structure are easy to understand if the uniform chain bands are redrawn, as in Fig. 4(a), in a reduced zone from $\Gamma$ to $Z=\pi / 4 d$ corresponding to four atoms per unit cell. The foldings cause the doubly degenerate $\pi$ band to appear at the reduced zone-edge, $Z$, where it has four-fold degeneracy, and is one half filled. As discussed herein, a small gap opens up at the zone edge due to Peierls distortion but can be safely ignored as it has no observable consequences. The computed bands for the $C$ structure are shown in Fig. 4(b). All bands below $E_{F}$ are nondegenerate. There are two bands which cross the Fermi level. It is noted that the bands in all optimized chain structures are similar, certainly as far as the number of bands crossing the Fermi level is concerned.

\section{CONDUCTANCE QUANTIZATION}

In 1957, Landauer ${ }^{23}$ introduced a novel way of looking at conduction as transmission. His famous formula was a major breakthrough in the conductance studies in mesoscopic systems and the concepts have been thoroughly discussed in several books. ${ }^{3-5}$ Based on the self-consistency arguments, namely the current flow changes the carrier density on two sides of the barrier, for a $1 \mathrm{D}$ conductor he derived the conductance $G_{b}=\left(2 e^{2} / h\right) T / R$, where $T$ and $R=1-T$ are transmission and reflection coefficients, respectively. This formula gives the intuitively appealing result that $G_{b} \rightarrow \infty$ as $R \rightarrow 0$, as one might expect in the absence of any scattering. The measured finite conductance arises by recognizing ${ }^{3}$ that this formula only gives the conductance of a barrier, $G_{b}$ in a 1D conductor and leaves out any contact effects. The conductance measured between two planes deep into the con- tacts within which the finite length conductor is placed is given $^{3-5,22-24}$ by $G=\left(2 e^{2} / h\right) T$. This includes the contact resistance $\left(h / 4 e^{2}\right.$ per contact) or the contacts conductance $G_{c}$ $=2 e^{2} / h$. We can readily verify that $G^{-1}=G_{c}^{-1}+G_{b}^{-1}$. Accordingly, even for perfect transmission, $T \sim 1$ the conductance is still finite and is equal to $2 e^{2} / h$. The corresponding resistance $R_{0}=G_{0}^{-1}=h / 2 e^{2}=12.9 \mathrm{k} \Omega$ is attributed to the resistance arising from the reflections at the contacts. It appears that the contact resistance is independent of the material or the nature of contact. Based on the work of Lang, ${ }^{16,17}$ we will remind the readers that this is not so.

As stated herein, the conductance per channel, $G$ $=\left(2 e^{2} / h\right) T$, and can have a maximum value of $G_{0}$ for an ideal ballistic channel. We can motivate this value of quantum conductance by appealing to the Heisenberg's uncertainty principle. ${ }^{14}$ Recalling that conductance $G=I / \Delta V$, and $I=\Delta Q / \Delta t$, then for a single channel in extreme quantum limit $\Delta Q=e$. One can readily write $G=e^{2} / \Delta E \Delta t$. Now invoking the uncertainty principle, $\Delta E \Delta t \geqslant h$, one gets $G$ $\leqslant 2 e^{2} / h$. Here, the factor of two is due to spin. The maximum conductance per channel or the channel capacity cannot exceed $G_{0}$.

One might ask if there is any justification for invoking the uncertainty principle. We believe that such a beautiful expression for conductance in terms of just two fundamental constants, $e$ and $h$, must have its origin in something rather fundamental. Even detailed 3D model potential calculations $^{25}$ end up with the same maximum channel capacity.

We attempt to justify that the invocation of the uncertainty principle is not just ad hoc. For this, we examine one particular derivation of the conductance formula for a constriction (of width $w$ and length $L$ ) in terms of density of states. For this quasi-1D system, the electrons are confined along $w$ (either by a gate voltage or by the physical termination of the sample) but behave as free electrons along $z$. One calculates the current density carried by each transverse channel and multiplies that with the number of channels $N \sim 2 w / \lambda_{F}$, where $w$ is the strip width transverse to the current flow direction. The current density ${ }^{4}$ in each channel, $J=e n_{s} V_{d}$ $=e n_{F} V_{F}$, involves either electron drift velocity $V_{d}$ or velocity $V_{F}$ associated with the channel at the Fermi energy. Here $n_{s}$ is the areal electron density and $n_{F}$ is a fraction thereof reduced by the factor $\left(V_{d} / V_{F}\right)$.

The number of carriers, $n_{F}$, which carry the current, moving at the Fermi velocity, can be expressed ${ }^{22}$ in terms of the 1D density of states, $\rho\left(E_{F}\right)$ at the Fermi energy for this channel. That is $n_{F}=\frac{1}{2}\left[\rho\left(E_{F}\right)\left(\mu_{1}-\mu_{2}\right) / w L\right]$, where $\mu_{1}$ and $\mu_{2}$ are the chemical potentials of the reservoirs between which the current flows. If one writes for the density of states (for $\pm p$ ), by recalling that each electron occupies a finite phase space,

$$
\rho(E) d E=2(\text { for } \operatorname{spin}) \frac{L 2 d p}{\Delta z \Delta p},
$$


where the other factor of two arises from the left- and righthand side moving electrons $( \pm p)$. The density of states at the Fermi energy is then simply

$$
\rho\left(E_{F}\right)=\frac{4 L}{\Delta z \Delta p V_{F}} .
$$

When we substitute this value in the expression for the total current $I$ the carrier velocity $V_{F}$ cancels out and

$$
I=J w=\frac{2 e\left(\mu_{1}-\mu_{2}\right)}{\Delta z \Delta p} .
$$

Further recognizing that $\mu_{1}-\mu_{2}=e \Delta V$,

$$
G=\frac{2 e^{2}}{\Delta z \Delta p} .
$$

It then becomes clear as to why it is possible to derive an expression for conductance using the uncertainty principle. The density of states itself embodies the uncertainty principle. The statement is sometimes made that the density of states drops out of the calculation. A more accurate statement would be that it is only the channel velocity that cancels out, but the factor $\Delta z \Delta p \sim h$ is still left behind.

Our interlude with the uncertainty principle not withstanding, it is well accepted ${ }^{16,17,22}$ that for 1D systems, each spin-degenerate band which crosses the Fermi level, provides one conduction channel which gives rise to $1 G_{0}$ $=\left(2 e^{2} / h\right)$ conductance if $T=1$. Accordingly, our band structure calculations project for the ideal ballistic conductance of 1D Al structures to be $2 G_{0}$. Lang ${ }^{16}$ performed elaborate first-principles calculations for the conductance of one to three $\mathrm{Al}$ atoms placed between two planar metallic electrodes. He reported values for resistance of 6.6, 9.0, and 8.3 $\mathrm{k} \Omega$ for wires consisting of 1,2 , and 3 atoms, respectively. For three atoms, the corresponding conductance is $1.6 G_{0}$. If we assume that three $\mathrm{Al}$ atoms represent a limiting value, then $T \simeq 0.8$ converts our ideal value to a more realistic one. This association is not nearly as clear cut as we have made it out to be. For one thing, Lang's calculation may involve a different number of conductance channels in the three atoms case than obtained in our periodic optimized zigzag chains. Also, the transmitivity is likely to depend on the nature of the channel.

Lang $^{17}$ also performed resistance calculations for $\mathrm{Na}$ atomic wires consisting of 1-4 $\mathrm{Na}$ atoms placed between macroscopic metallic electrodes. The resistance values for $\mathrm{Na}$ he obtained are $33,16.5,19$, and $17.5 \mathrm{k} \Omega$ for wires consisting of 1, 2, 3, and 4 atoms, respectively. These resistance values are large compared to $\mathrm{Al}$ wires. One reason for this is that $\mathrm{Na}$ has only one conduction channel. The conductance values for $\mathrm{Na}$ atomic wires are all fractional when expressed in terms of $G_{0}$. It is 0.4 for one atom and rises to 0.8 for two atoms and essentially saturates there, again suggesting an average $T \simeq 0$.8. Resistance values for single atoms of $\mathrm{Al}(6.6$ $\mathrm{k} \Omega)$ and $\mathrm{Na}(33 \mathrm{k} \Omega)$ placed between electrodes are wildly different. This shows that contact resistance, which dominates the one atom case, is highly material and contact specific. These differences are easy to reconcile in terms of the resonant tunneling calculations of Kalmeyer and Laughlin. ${ }^{25}$ They find that the maximum value of the (differential) conductance on resonance is indeed $G_{0}$ for all tunnel barriers. The resonance, in general, will not coincide with the Fermi level, and thus depending on the atom different values can be explained.

\section{WIEDEMANN-FRANZ LAW IN NANOWIRES}

In order to derive the Wiedemann-Franz law for nanowires, we must first calculate the thermal conductance arising from the thermal energy transported by electrons as they move between reservoirs held at different temperatures. An important observation in this regard is that the quantum of thermal conductance can also be obtained (within a numerical factor) as a consequence of the Heisenberg's uncertainty principle. ${ }^{15}$ One might argue that this indeed should be the case since the current carrying channels are also the ones that transport the thermal energy. This is essentially correct, except that for thermal conductance there should be no net electric current. Thus, an electron moving from a reservoir at a higher temperature must be compensated ${ }^{26}$ by a colder electron.

The steady state heat current density (i.e., heat transfer per unit time per unit cross section) in a diffused regime in one dimension is given ${ }^{27,26}$ by $j_{H}=-K d T / d x$, in terms of the temperature gradient and the thermal conductivity $K$. For a quasi-1D metallic nanowire connecting two reservoirs (i.e., left-hand side reservoir $L$ and right-hand side reservoir $R$ ) the energy transported by electrons dominates over that transported by phonons. Under ballistic conditions in the absence of any net current, the heat current (or the flux of electronic thermal energy) from $L$ to $R, \mathcal{J}_{e}$ depends only on the temperature difference between two reservoirs, $\Delta T=T_{L}-T_{R}$.

The thermal conductance $\mathcal{K}_{e}$ is the ratio of the total electronic heat current to the temperature difference,

$$
\mathcal{K}_{e}=\frac{\mathcal{J}_{e}}{\Delta T} .
$$

Recalling that the total heat current $\mathcal{J}_{e}=d E / d t$, and when a single carrier moves from $L$ to $R$ across the wire, an energy $k_{B} T_{L}$ flows across the junction. In this limit, one can also assume that the transit time to lie in the range set by the Heisenberg's uncertainty principle $(d t \sim \Delta t)$, but to maintain the zero-current condition, one carrier must also flow from $R$ to $L$ across the nanowire. Hence under ballistic conditions $d E=k_{B} \Delta T$ and

$$
\mathcal{J}_{e}=\frac{k_{B} \Delta T}{\Delta t} .
$$

Assuming the uncertainty in energy to be of the order of thermal spread, $\Delta E \sim k_{B} T$, where $T=\left(T_{L}+T_{R}\right) / 2$, and combining Eqs. (5) and (6), one gets

$$
\mathcal{K}_{e} \sim \frac{k_{B}^{2} T}{\Delta E \Delta t} .
$$

Now, invoking the Heisenberg's uncertainty leads to 


$$
\mathcal{K}_{e} \sim 2 \frac{k_{B}^{2}}{h} T,
$$

where the factor of two arises from the spin degeneracy. This expression may well be off by factors of $2, \pi$, etc. The precise expression for the quantum of thermal conductance is $^{26} \mathcal{K}_{o}=\left(\pi^{2} / 3\right)\left(k_{B}^{2} / h\right) T$. If we now construct the ratio of $\mathcal{K}_{e} / G_{0}$, we get

$$
W=\frac{\mathcal{K}_{e}}{G_{0}}=\frac{k_{B}^{2} T}{e^{2}} .
$$

This, apart from a numerical factor, is the statement of the Wiedemann-Franz law. ${ }^{26,27}$ It is fascinating that it holds equally well in the quantum domain.

\section{SUMMARY}

First-principles based structural arrangement of atoms for 1D structures and the resultant electronic structure enable a projection for the ideal quantum ballistic transport using channel capacity arguments. Aluminum atoms may form a zigzag chain or dual dumbbells $C$ structure in quasi-1D system leading to a maximum of $4 e^{2} / h$ conductance. The channel capacity is motivated using the uncertainty principle. Similar arguments support that the Wiedemann-Franz law holds in the nanodomain.

Presented at the IUVSTA 15th International Vacuum Congress and the AVS 48th International Symposium, and the 11th International Conference on Solid Surfaces, San Francisco, CA, 28 October-2 November 2001.

${ }^{1}$ B. J. van Wees, J. van Houten, C. W. J. Beenackker, J. G. Williams, L. P. Kouwenhowen, D. van der Marel, and C. T. Foxon, Phys. Rev. Lett. 60, 848 (1988).

${ }^{2}$ D. A. Wharam, T. J. Thornton, R. Newbury, M. Pepper, H. Ritchie, and
G. A. C. Jones, J. Phys.: Condens. Matter 21, L209 (1988).

${ }^{3}$ Y. Imry, in Directions in Condensed Matter Physics, edited by G. Grinstein and G. Mazenko (World Scientific, Singapore, 1986), p. 101.

${ }^{4}$ S. Datta, Electronic Transport in Mesoscopic Systems (Cambridge University Press, Cambridge, UK, 1985).

${ }^{5}$ Y. Imry, Mesoscopic Physics (Oxford University Press, Oxford, 1997).

${ }^{6}$ G. Binning, H. Rohrer, Ch. Gerber, and E. Weibel, Phys. Rev. Lett. 49, 57 (1982).

${ }^{7}$ J. K. Gimzewski and R. Möller, Phys. Rev. B 41, 2763 (1987).

${ }^{8}$ N. Agraït, J. G. Rodrigo, and S. Vieria, Phys. Rev. B 47, 12345 (1993).

${ }^{9}$ H. Ohnishi, Y. Kondo, and K. Takayanagi, Nature (London) 395, 780 (1998).

${ }^{10}$ A. I. Yanson, G. R. Bollinger, H. E. Brom, N. Agrait, and J. M. Ruitenbeek, Nature (London) 395, 783 (1998).

${ }^{11}$ D. S. Portal, E. Atracho, J. Junquera, P. Odrejón, A. García, and J. Soler, Phys. Rev. Lett. 83, 3884 (1999); also see, H. Häkkinen, R. N. Barnett, and U. Landman, J. Phys. Chem. B 103, 8814 (1999).

${ }^{12}$ D. S. Portal, E. Atracho, J. Junquera, A. García and J. Soler (arXiv:condmat/0010501)

${ }^{13}$ P. Sen, S. Ciraci, A. Buldum, and I. P. Batra, Phys. Rev. B 64, 195420 (2001).

${ }^{14}$ I. P. Batra, Surf. Sci. 395, 43 (1998).

${ }^{15}$ S. Ciraci, A. Buldum, and I. P. Batra, J. Phys.: Condens. Matter 13, R537 (2001).

${ }^{16}$ N. D. Lang, Phys. Rev. B 52, 5335 (1995).

${ }^{17}$ N. D. Lang, Phys. Rev. Lett. 79, 1357 (1997).

${ }^{18}$ D. Vanderbilt, Phys. Rev. B 41, 7892 (1990); G. Kresse and J. Hafner, J. Phys.: Condens. Matter 6, 8245 (1994).

${ }^{19}$ G. Kresse and J. Hafner, Phys. Rev. B 47, R558 (1993); G. Kresse and J. Furtmüller, ibid. 54, 11169 (1996).

${ }^{20}$ R. O. Jones, J. Chem. Phys. 99, 1194 (1993); B. K. Rao and P. Jena, ibid. 111, 1890 (1999).

${ }^{21}$ O. Gulseren, F. Ercolesi, and E. Tosatti, Phys. Rev. Lett. 80, 3775 (1998).

${ }^{22}$ M. Brandbyge, K. W. Jacopsen, and J. K. Nørskov, Phys. Rev. B 52, 8499 (1995); 55, 2637 (1997); 56, 14956 (1997).

${ }^{23}$ R. Landauer, IBM J. Res. Dev. 1, 223 (1957); Philos. Mag. 21, 863 (1970); J. Phys.: Condens. Matter 1, 8099 (1989).

${ }^{24}$ M. Büttiker, Y. Imry, R. Landauer, and S. Pinhos, Phys. Rev. B 31, 6207 (1985).

${ }^{25}$ V. R. Kalmeyer and R. B. Laughlin, Phys. Rev. B 35, 9805 (1987).

${ }^{26}$ J. M. Ziman, Theory of Solids (Cambridge University Press, Cambridge, UK, 1965), p. 196.

${ }^{27}$ C. Kittel, Introduction to Solid State Physics (Wiley, New York, 1996), p. 166. 